\title{
Electrohydraulic Proportional Metering Valve for Common Rail System
}

\author{
Sonam YUN*, Jae Seop RYU*, Byoung Kyu AHN*, Go Do KIM** and Jung Hyun JO** \\ * Intelligence and Precision Machinery Research Division, \\ Korea Institute of Machinery and Materials(KIMM) \\ 171 Jang-dong, Yuseong-gu, Daejeon, 305-343 Korea \\ (E-mail: ysn688@kimm.re.kr) \\ ** Technology Institute, SG Servo Inc. \\ 57-6 Palyong-dong, Chanwon, Kyungnam, 641-846 Korea
}

\begin{abstract}
In the common rail system with direct injection equipment in diesel engine, a proportional metering valve to control the fuel flow into the high pressure pump is used. The proportional solenoid actuator is suitable for metering valve because it has a constant attraction force in the control region independently on the stroke position and a fast motion response of the plunger. The force characteristics with respect to the stroke of the proportional solenoid actuator is strongly depending on the shape of the control cone, but it is difficult to design the control cone. In this paper, a proportional solenoid actuator of electrohydraulic proportional metering valve for common rail system was analyzed using finite element method and the shape of the control cone of the proportional solenoid actuator was optimized to get the constant force in the control region. The analysis results of the optimized proportional solenoid actuator were compared with the experimental results of the manufactured one.
\end{abstract}

\section{KEY WORDS}

Common rail system, Proportional solenoid, Proportional metering valve, PWM control, Fuel injection

\section{NOMENCLATURE}

$L$ : Control cone length

$t_{c}:$ Control cone thickness

$W$ : Width between plunger and bobbin

$G_{a p}: \quad$ Gap between plunger and control cone

\section{INTRODUCTION}

This study deals with a low pressure proportional metering valve with a proportional solenoid actuator for a common rail system of the diesel engine vehicle. This actuator is installed at the inlet part of the high pressure fuel pump and has the function of controlling the flow rate from the low pressure fuel pump. This fuel flow is controlled in proportion to the engine RPM. So this control operation enables the engine to inject the optimal of fuel. Generally, diesel vehicles emit the toxic gases including nitrogen and produce noise during operation. These problems deteriorate the comport of passengers, environmental pollution and energy saving, for which a improvement has been required. As the solution for the above-mentioned problems, the method, which involves the injection of high pressure and atomization of fuel, was developed to improve the 
combustion process of diesel engines used in vehicles. It is also very important to optimally control the supply of the initial amount of fuel for atomization. However, there is further need for the optimal design of a low pressure proportional solenoid actuator[1][2].

This is a basic study on an optimal method of fuel injection and a low pressure proportional metering valve with a solenoid actuator[3][4][5].

Figure 1 shows a common rail system with a low pressure proportional metering valve with a solenoid actuator inside, which is the object of this study.

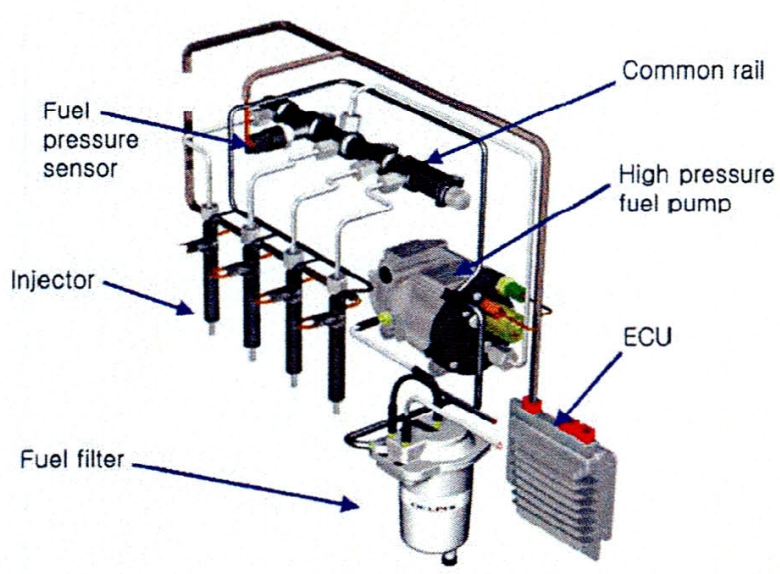

Figure 1 Common rail system of diesel engine

\section{DESIGN OF PROPORTIONAL SOLENOID ACTUATOR}

Figure 2 shows a low pressure proportional metering valve for a common rail, consisting of a coil, stationery core, plunger and poppet type flow control valve.

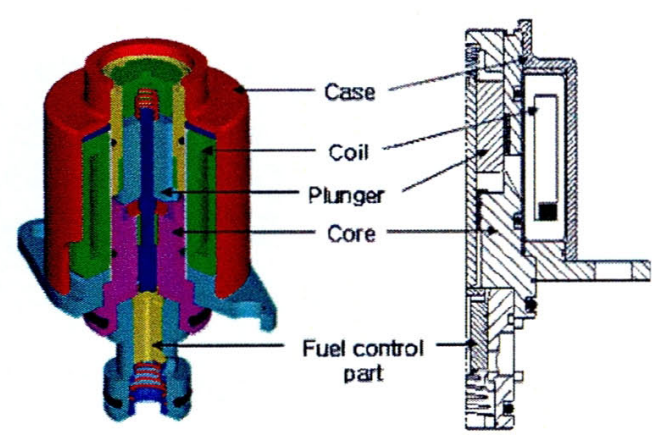

Figure 2 proportional metering valve for common rail

\section{$2.11^{\text {st }}$ design}

Figure 3 shows the proposed solenoid actuator in the first step of concept design. The control cone of the proportional solenoid plays a vital role of controlling attraction force in proportion and its properties have to be understood through analysis. During the step of concept design, we conducted the analysis by inputting the design factors in Table 1 into the drawing of Figure 3. The specifications were input voltage $12[\mathrm{~V}]$, input current $1.1[\mathrm{~A}]$, and attraction force $10[\mathrm{~N}]$.

The a) in Figure 4 shows the experimental results when there is almost no gap between the control cone and the plunger and the thickness of the control cone, $t_{\mathrm{c}}$, was fixed at $1.5 \mathrm{~mm}$. It was observed that the closer the plunger came to the stationary core, the lower the attraction force was. The b) of Figure 4 shows the experimental results when the thickness of the control cone was fixed at $0.5 \mathrm{~mm}$ but there was no visible effect. We believe that it is resulted from the too-large magnetic flux leakage generated because there was no gap between the plunger and the control cone and because the width of the control cone, $W$, was very large[6][7][8].

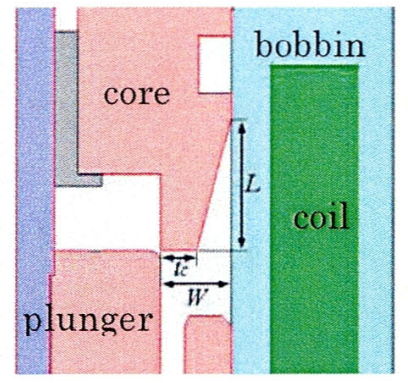

Figure 3 Analysis model_1

Table 1 Design parameters for model_1

\begin{tabular}{|c|c|c|}
\hline Item & Model 1 & Model 1(new) \\
\hline$W$ & $3.0 \mathrm{~mm}$ & $3.0 \mathrm{~mm}$ \\
\hline$L$ & $5.6 \mathrm{~mm}$ & $5.6 \mathrm{~mm}$ \\
\hline$t_{c}$ & $1.5 \mathrm{~mm}$ & $0.5 \mathrm{~mm}$ \\
\hline
\end{tabular}

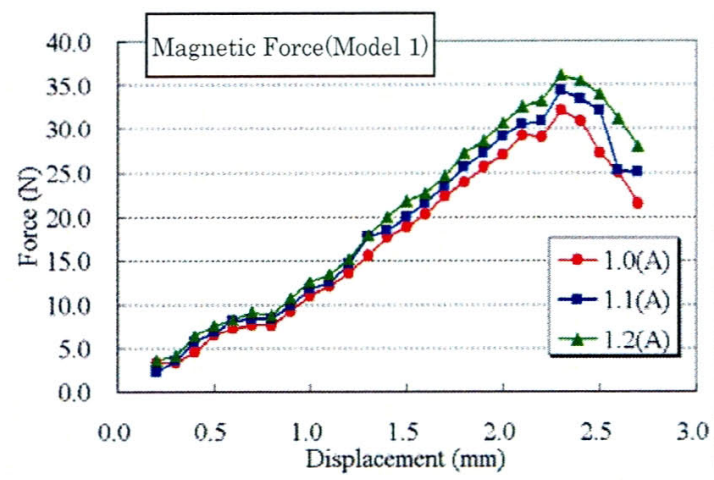

a) Model_1 


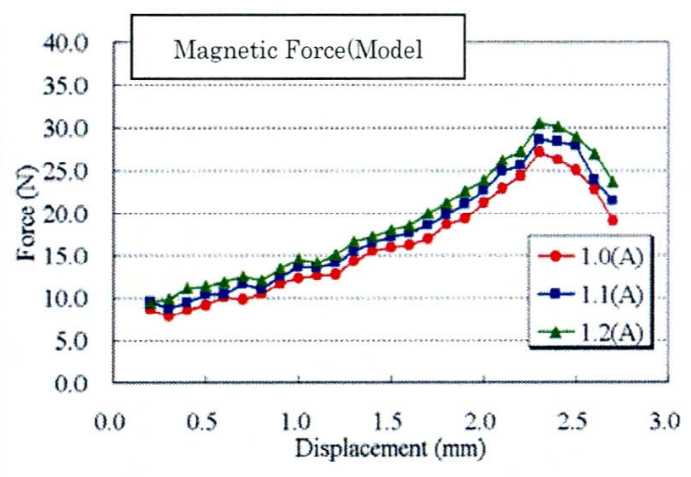

b) Model_1(new)

Figure 4 Analysis result of model_1

\section{$2.22^{\text {nd }}$ design}

Figure 5 shows new proposed models for compensating the attraction force shown in Model 1. Both a) and b) in Figure 5, are the experiment results, when conducted with the reduced values of control length, $L$, and width, $W$, compared to those of Model_1. And the b) in Figure 5 is the result when conducted with the width, $W$, of less than $1 \mathrm{~mm}$ compared to a) in order to reduce magnetic flux leakage. It was confirmed that b) in Figure 6 has more excellent properties than a) However, the shape of the sealing part was not good in effectively preventing leakage and an optimal design technique is needed.

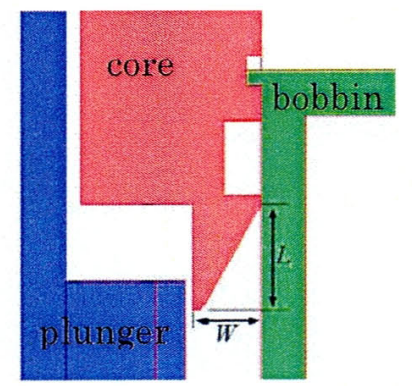

a) Analysis model_2(L:3.5mm, $W: 2.25 \mathrm{~mm})$

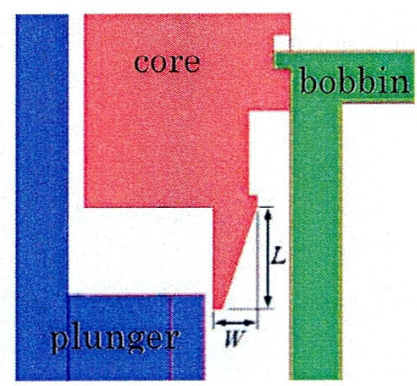

b) Analysis model_3(L:3.0mm, W: $1.25 \mathrm{~mm})$

Figure 5 Proposed model_2 and model_3

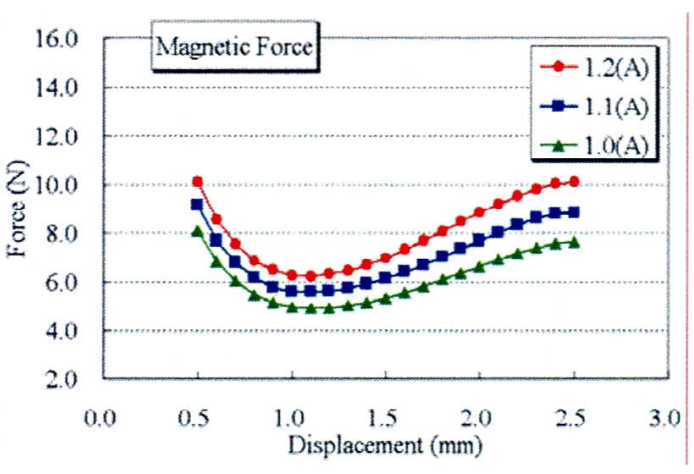

a) Model_2

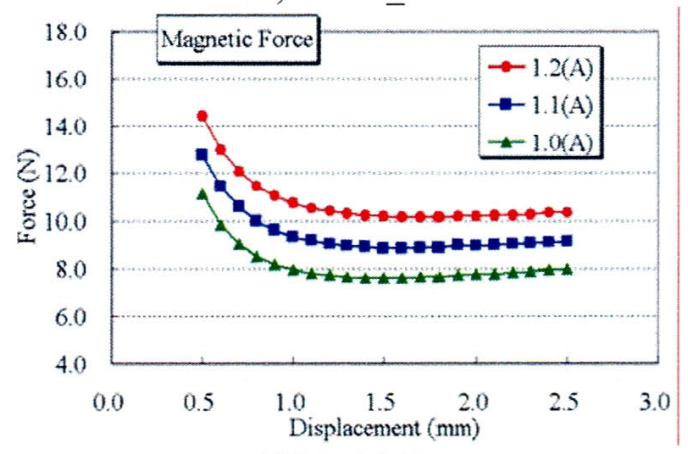

b) Model_3

Figure 6 Analysis results of model_2 and model_3

\section{$2.33^{\text {rd }}$ design}

Figure 7 is designed to provide an optimal solenoid actuator from the shape in Figure 5, which manufacturing condition and seal property were taken into consideration.

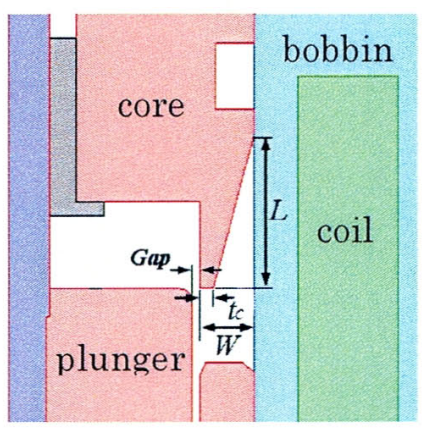

Figure 7 Final model for proportional solenoid actuator

Table 2 Design parameters for final model

\begin{tabular}{|c|c|c|c|}
\hline Item & Model 2-1, 2-2,2-3 & Model 2-4 & Model 2-5 \\
\hline$W$ & $2.0 \mathrm{~mm}$ & $1.0 \mathrm{~mm}$ & $2.0 \mathrm{~mm}$ \\
\hline$L$ & $5.6 \mathrm{~mm}$ & $3.2 \mathrm{~mm}$ & $5.6 \mathrm{~mm}$ \\
\hline$t_{c}$ & $0.5 \mathrm{~mm}$ & $0.5 \mathrm{~mm}$ & $0.25 \mathrm{~mm}$ \\
\hline$G_{a p}$ & $0.25 \mathrm{~mm}$ & $0.25 \mathrm{~mm}$ & $0.25 \mathrm{~mm}$ \\
\hline
\end{tabular}


Figure 8 shows the finite element results analyzed by inputting each design factors shown in Table 2 . It was confirmed that in the case of $W=2.0 \mathrm{~mm}, L=5.6 \mathrm{~mm}, t_{c}=$ $0.25 \mathrm{~mm}, G_{a p}=0.25 \mathrm{~mm}$, high attraction force was gotten and was constantly controlled regardless of changes of plunger stroke.

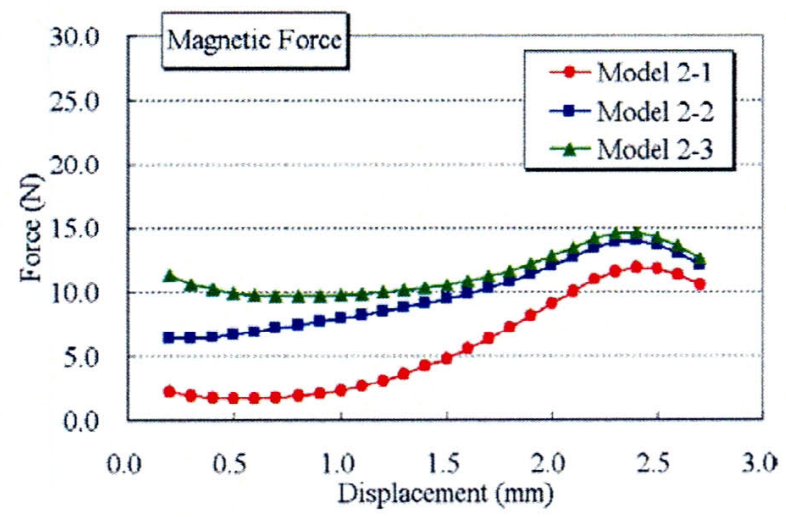

a) Model 2-1, 2-2, 2-3

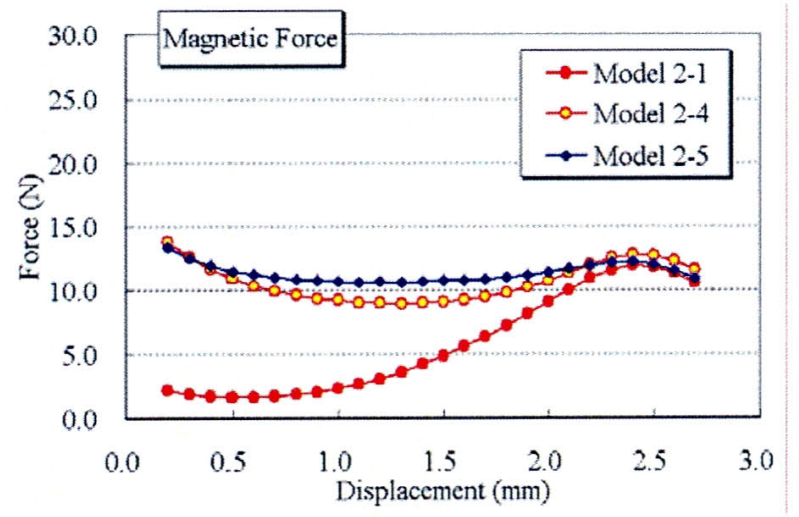

b) Model 2-1, 2-4, 2-5

Figure 8 Analysis results of final models

\section{EXPERIMENTAL RESULTS}

\subsection{Experimental apparatus}

We made a prototype proportional metering valve with a solenoid actuator based on the analysis results in Table 2 and Figure 8, and in order to verify the actuator's performance, we composed the experimental apparatus shown in Figure 9. And then observed the flow and pressure property of the solenoid actuator from the experimental apparatus. To input the signals into the solenoid, we used the PWM controller presently embedded in leisure vehicles. Figure 10 is the photo view of the experimental apparatus.

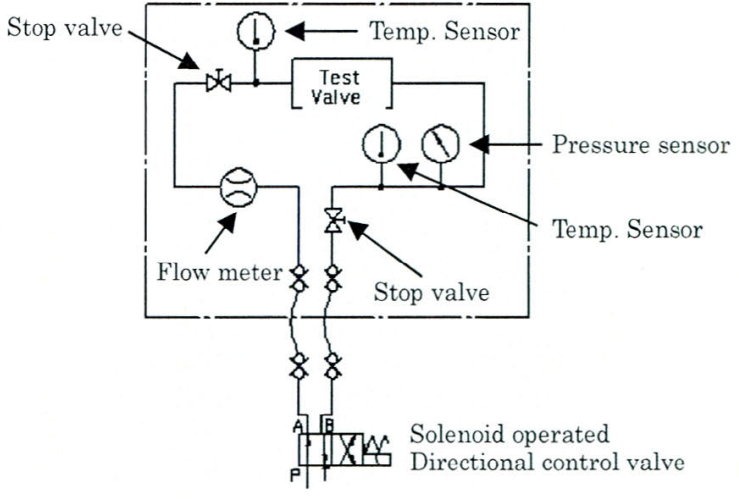

Figure 9 Hydraulic circuit of experimental apparatus

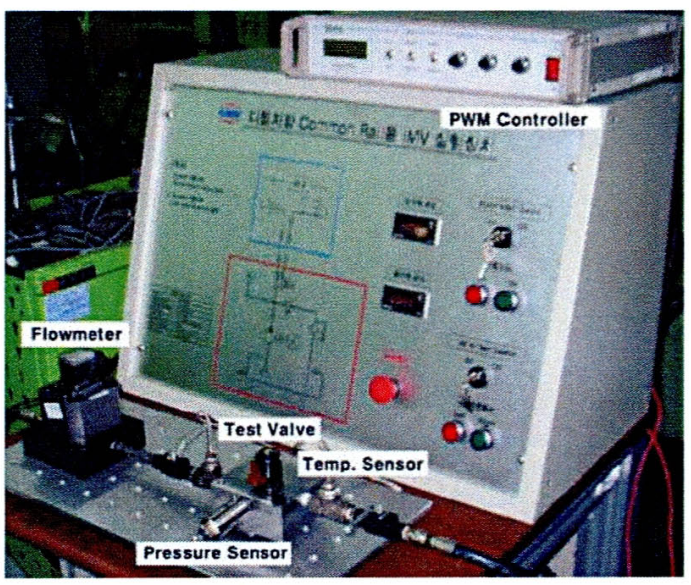

Figure 10 Photo view of experimental apparatus

\subsection{Experimental results}

Figure 11 shows the experimental results of the prototype. after setting any number of frequency inputted into the proportional solenoid actuator, these are the results of the pressure of the valve and the flow in accordance with the manipulation of duty ratio. First, the noise was the least at the $1000 \mathrm{~Hz}$ PWM signal input, but flow and pressure hysteresis were most excellent at the $400 \mathrm{~Hz}$ PWM signal input. Because the reduction of the causes of noise to improve comport is an important factor in designing a vehicle, there is a need to design a valve that has a good performance at high frequencies. We believe that a valve precision manufacturing can easily solve the problem.

Figure 12 shows the experimental results of the 2 nd prototype. The 2nd prototype was precisely manufactured with better machinability, which was a trouble of the 1st prototype. Therefore, hysteresis at the high frequencies was improved, which was a problem of the 1 st prototype. 


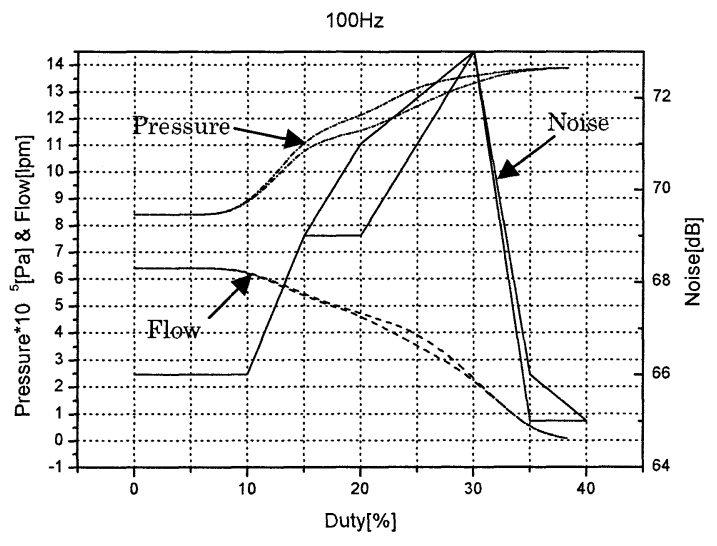

a) $100 \mathrm{~Hz}$ PWM signal input

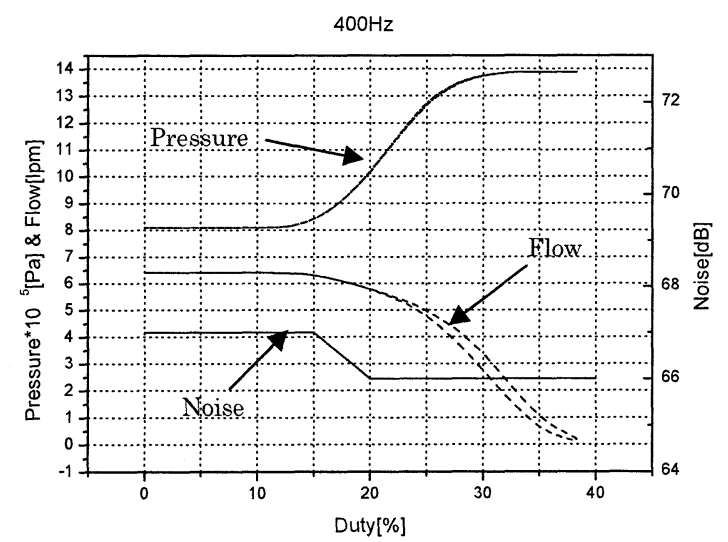

b) $400 \mathrm{~Hz}$ PWM signal input

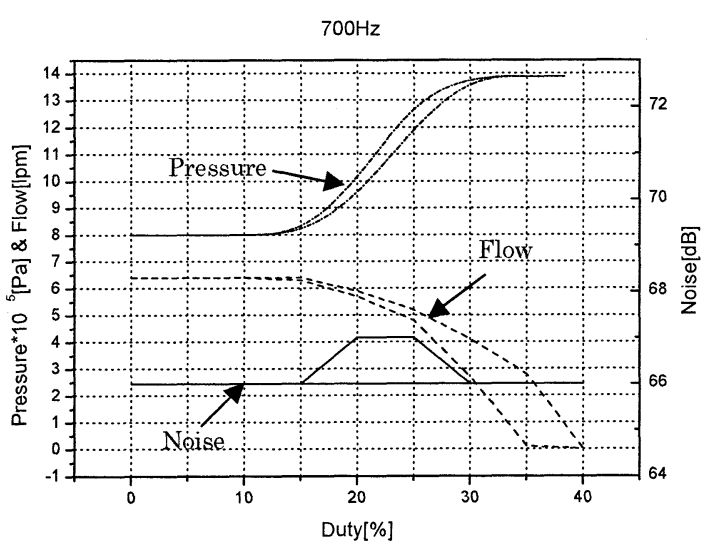

c) $700 \mathrm{~Hz}$ PWM signal input

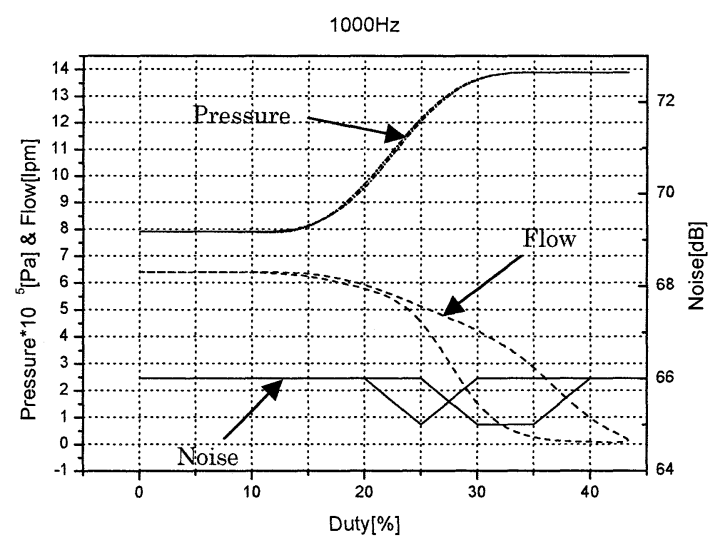

d) $1000 \mathrm{~Hz}$ PWM signal input

Figure 11 Experimental results of 1st prototype

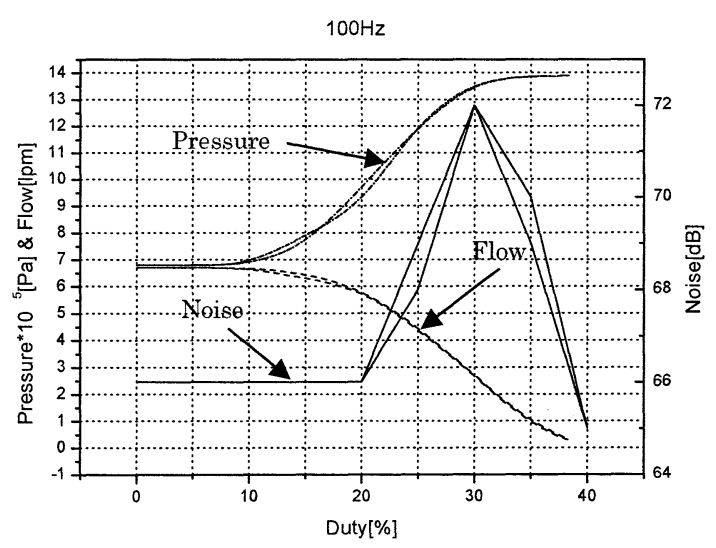

a) $100 \mathrm{~Hz}$ PWM signal input

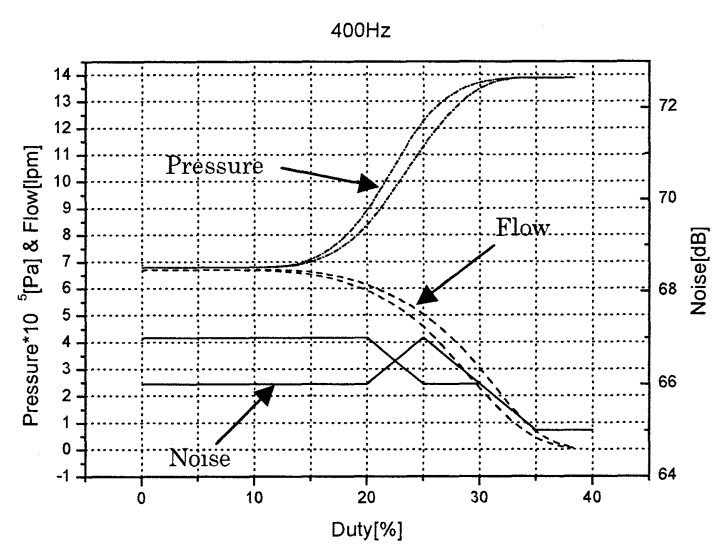

b) $400 \mathrm{~Hz}$ PWM signal input 


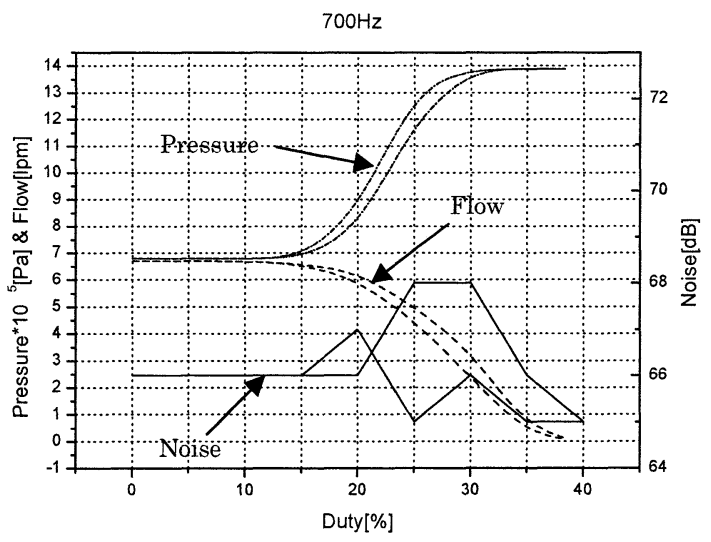

c) $700 \mathrm{~Hz}$ PWM signal input

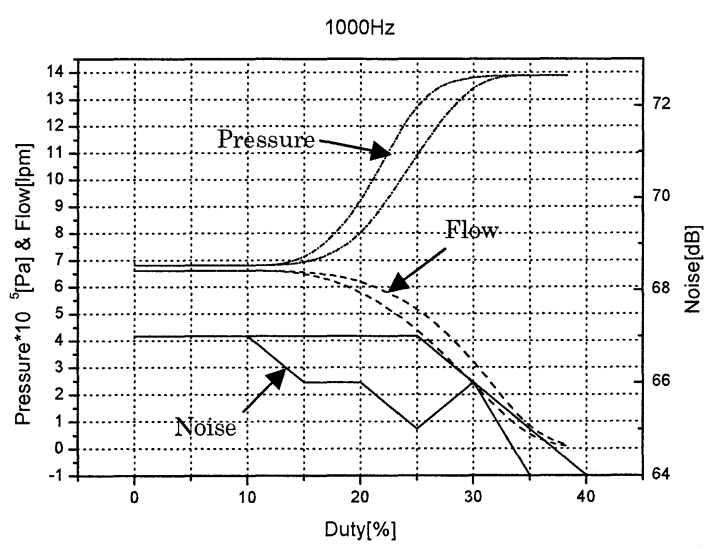

c) $1000 \mathrm{~Hz}$ PWM signal input

Figure 12 Experimental results of 2nd prototype

\section{CONCLUSION}

In this study, we conducted an analysis of the electromagnetic and design of a low pressure proportional metering valve with a solenoid actuator from a leisure disel vehicle applied to a common rail system, and we verified its performance by manufacturing it. The comparative factors such as noise, flow and pressure hysteresis depend on the given conditions of the PWM signal. It was verified that these problems could be easily solved through a precision process. In addition, the results of this study can be utilized in developing and improving the proportional metering valve with a solenoid actuator for the common rail system.

\section{REFERENCES}

1. Bruno P. Lequesne, Finite Element Analysis of a Constant-Force solenoid for Fluid Flow Control,
IEEE, Tran. Industrial Application, 1988, 24-4, pp.574-581.

2. Grit Geiler, Modellbildung von Proportional magneten mittels konzentrierter Parameter, $+\mathrm{P}, 2001$, 45-1, pp.49-52.

3. C.S. Koh, J.O. Kim, S.Y. Hahn and Osama. A. Mohammed, Optimum design of Voice Coil Motor with constant torque coefficients using evolution strategy, Journal of Applied Physics, 1994, 75-10, pp.6045-6047.

4. Sonam Yun, Kyungwoo Lee and Honghee Kim, Development of the Pneumatic Valve with Birmorph Type Piezoelectric Actuator, 1st International Workshop on Piezoelectric Materials \& Applications in Actuator, 2004, p.118.

5. Jae Seop Ryu, Mi Ran Seo, Chang Seop Koh, Sonam Yun and Dong Soo Kim, Optimal Design of Constant-Force Solenoid for Pressure Control Using Axisymmetric Non-linear Parameterized Sensitivity Analysis, The 11th Biennial IEEE Conference on Electromagnetic Field Computation, 2004, p.390.

6. Sonam Yun, Electro-Magnetic Proportional Solenoid, JFPS, 2004, 35-5, pp.342-347.

7. Sonam Yun, Young-Bog Ham, Characteristics analysis of the solenoid valve for exhaust brake, Transactions of KSAE, 2005, 12-1, pp.190-195.

8. Sonam Yun, Jae-seop Ryu, Byung-Kyu Ahn, Mi-ran Seo and Chang-seop Koh, Optimal design of electro-magnetic proportional solenoid using genetic algorithm, Proceeding of the 50th national conference on fluid power, 2005, pp.243-247. 